\section{Commentary on the identity of fibroblast pneumocyte factor: rat vs. human}

Advance online publication, 31 May 2017; doi:10.1038/pr.2017.85

To the Editor: Fetal lung maturation is accelerated by increasing levels of endogenous glucocorticoids in late gestation and by exogenous corticosteroid treatment in many species, including humans, in whom antenatal corticosteroid therapy is standard of care for premature infants. In their recent review, King et al. (1) addressed the mechanisms involved in accelerated appearance of fetal lung type 2 pneumocytes and pulmonary surfactant with glucocorticoid treatment and stated that "this stimulatory effect is indirect." They reviewed the extensive evidence on the role of lung fibroblasts and release of glucocorticoid-induced peptide factor(s) termed fibroblast-pneumonocyte factor (FPF) that acts on adjacent epithelial cells to augment the synthesis of surfactant phospholipids and proteins.

It is important to note, however, that the majority of evidence for FPF is in rats and relates to the synthesis of disaturated phosphatidycholine (DSPC) as measured by incorporation of labeled choline. The response for choline incorporation by fetal rat lung epithelial cells, which were isolated from cortisol-treated organotypic cultures, to partially purified FPF was rapid and consistent with posttranslational activation of choline-phosphate cytidylyltransferase contributing to increased PC production (2). In our earlier studies with human fetal lungs, dexamethasone stimulation of choline incorporation into DSPC was similar with explants $(\sim 50 \%$ fibroblasts) and isolated epithelial cells ( $10 \%$ fibroblasts), suggesting little apparent dose-response effect for fibroblast content (3). In human epithelial cells, dexamethasone (in the presence of cyclicAMP) increased mRNA for a total of 13 genes related to lipid uptake, biosynthesis, and remodeling, indicating that induction of surfactant DSPC production and lamellar body formation likely involves transcriptional regulation of more proteins than those that participate in the choline incorporation pathway (4). Additional studies in human fetal lungs, including effects of fibroblast-conditioned medium and characterization of regulated expression of lipogenic proteins, are needed to determine the possible involvement of FPF in human lung DSPC synthesis.

Other studies with human fetal lungs indicate that glucocorticoid stimulation of some key surfactant-related genes most likely occurs as a result of glucocorticoid effects directly in lung epithelial cells. On using isolated epithelial cells of second-trimester human fetal lungs, which are
90-95\% pure, differentiation into type 2 cells occurs with glucocorticoid treatment, which is augmented by the presence of cyclicAMP. The observed responses include morphological differentiation with reduced glycogen and appearance of lamellar bodies, induction of a variety of genes related to surfactant and other type 2 cell-specific functions, and stimulated synthesis and secretion of DSPC from cells (3). Induction of several surfactant-related genes is mediated in full (eg., LAMP3, a lamellar body protein, and CEACAM6, a surfactant-binding protein) or in part (SFTPA, SFTPB, SFTPC, ABCA3) by increased content of nuclear thyroid transcription factor 1 (TITF1, NKX2-1), a key transcription factor in type 2 cell differentiation. Consistent with a requirement for induction of TITF1, the transcription rate for CEACAM6 does not increase until $7-12 \mathrm{~h}$. TITF1 is expressed in epithelial cells but not in fetal fibroblasts of human fetal lung; glucocorticoids maximally increase TITF1 mRNA within $4 \mathrm{~h}$ and this response is not blocked by an inhibitor of protein synthesis (cycloheximide) as occurs for FPF in rat lung (5). Increased transcription of SFTPB, a type 2 cell-specific protein that is critical for surfactant function, occurs within $2 \mathrm{~h}$ and is not blocked by cycloheximide (6). A mechanistic model of glucocorticoid induction involving a fibroblast factor would presumably involve the binding of glucocorticoid to fibroblast glucocorticoid receptors, translocation of the complex to the nucleus, induction, synthesis, and secretion of a mediating protein(s), binding (or uptake) of the protein in epithelial cells, and transcriptional stimulation, directly or indirectly, of glucocorticoid target genes or posttranslational activation of proteins-a process that would require many hours. Thus, at least for TITF1 and SFTPB, which have been studied in some detail, the rapidity of the responses and independence from induction of a mediating protein are not consistent with glucocorticoid effects via fibroblast-derived factor(s) in human lungs. A caveat to these observations is the current lack of evidence for a functional glucocorticoid response element in surfactant protein genes, perhaps implying a complex regulatory mechanism within type 2 cells.

King et al. (1) discuss three proteins as potential candidates for FPF activity observed in rat lung: KGF (FGF7), leptin (LEP), and neuregulin (NRG1). Recently, we performed RNAseq analysis of human fetal lung (23-24 wk) tissue before (preculture) and after culture as explants in the presence or absence of budesonide, a potent synthetic glucocorticoid (7). Explants are prepared by mincing tissue into $\sim 1 \mathrm{~mm}^{3}$ pieces that are cultured in serum-free medium. Explants contain all lung-cell types, predominantly epithelial cells and fibroblasts, and thus mimic the in vivo situation. Results for gene expression of the putative FPF proteins and selected receptors are shown in Table 1. In preculture lung, 
Table 1. Gene expression of putative FPF proteins and receptors in human fetal lung by RNAseq

\begin{tabular}{lcc}
\hline Gene & $\begin{array}{c}\text { Preculture mRNA } \\
(\mathrm{cpm}) \text { mean } \pm \text { SD }\end{array}$ & $\begin{array}{c}\text { Explant culture } \\
\text { (budesonide/control) }\end{array}$ \\
\hline LEP & $<1$ & - \\
NRG1 & $3.8 \pm 1.6$ & 0.71 \\
FGF7 & $92.3 \pm 12.1$ & $0.26^{\mathrm{a}}$ \\
LEPR & $33.8 \pm 5.6$ & 0.99 \\
LEPROT & $167.3 \pm 5.1$ & 1.06 \\
ERBB3 & $272.0 \pm 37.4$ & 1.23 \\
ERBB4 & $5.5 \pm 2.9$ & 1.40 \\
FGFR2 & $136.8 \pm 10.6$ & 1.28 \\
\hline
\end{tabular}

cpm, counts per million; FPF, fibroblast-pneumonocyte factor.

${ }^{\text {a }}$ False discovery rate $=0.02$.

RNAseq data from three lungs of 23-24 wk gestation before and after culture for

4 days with/without $30 \mathrm{nM}$ budesonide as described (7).

leptin mRNA is not detected, NRG1 mRNA is present in low amounts, and there are abundant FGF7 transcripts; expression of receptors for each protein was found. LEP mRNA was also not detected in earlier gene profiling of human fetal lung epithelial cells cultured with/without glucocorticoid (7); these negative results for LEP expression in mid-gestation human fetal lung are similar to those for late-gestation fetal lamb and contrast with those for late-gestation rat (1). Culture of explants with budesonide for 4 days did not induce any of the genes and significantly repressed FGF7 4-fold, while a number of known glucocorticoid-responsive genes were regulated by budesonide (7). By contrast, as reviewed by King et al. (1), dexamethasone increases mRNA for both FGF7 and leptin in fetal rat lung fibroblasts, consistent with actinomycin sensitivity of FPF induction, while dexamethasone does not increase NRG1 mRNA in rat fibroblasts, suggesting that NRG1 is not FPF.

Thus, studies in human lungs suggest that many glucocorticoid effects on type 2 cell differentiation and surfactant production occur within epithelial cells, independent of an induced factor in fibroblasts. In addition, there are apparent species differences for LEP expression in fetal lung tissue of humans and rats, perhaps on a developmental basis. The RNAseq data do not support a role for induction of FGF7, LEP, or NRG1 in mediating glucocorticoid effects in cultured human fetal lungs. These observations suggest caution in attempting to extrapolate findings in rats to humans, particularly with regard to potential therapeutic approaches to enhance lung maturity in premature infants.

\section{STATEMENT OF FINANCIAL SUPPORT \\ This study was supported by NHLBI 1R21HL129910-01. \\ Disclosure: The author declares no conflict of interest.}

\section{Philip L. Ballard ${ }^{1}$}

${ }^{1}$ Department of Pediatrics University of California, San Francisco, California

\section{Correspondence: Philip L. Ballard (Phil.ballard@ucsf.edu)}

\section{REFERENCES}

1. King G, Smith ME, Cake MH, Nielsen HC. What is the identity of fibroblast-pneumocyte factor? Pediatr Res 2016;80:768-76.

2. Post M, Barsoumian A, Smith BT. The cellular mechanism of glucocorticoid acceleration of fetal lung maturation. Fibroblastpneumonocyte factor stimulates choline-phosphate cytidylyltransferase activity. J Biol Chem 1986;261:2179-84.

3. Gonzales LW, Guttentag SH, Wade KC, Postle AD, Ballard PL. Differentiation of human pulmonary type II cells in vitro by glucocorticoid plus cAMP. Am J Physiol Lung Cell Mol Physiol 2002;283: L940-51.

4. Wade KC, Guttentag SH, Gonzales LW, et al. Gene induction during differentiation of human pulmonary type II cells in vitro. Am J Respir Cell Mol Biol 2006;34:727-37.

5. Kolla V, Gonzales LW, Gonzales J, et al. Thyroid transcription factor in differentiating type II cells: regulation, isoforms, and target genes. Am J Respir Cell Mol Biol 2007;36:213-25.

6. Ballard PL, Ertsey R, Gonzales LW, Gonzales J. Transcriptional regulation of human pulmonary surfactant proteins SP-B and SP-C by glucocorticoids. Am J Respir Cell Mol Biol 1996;14:599-607.

7. Barrette AM, Roberts JK, Chapin C, et al. Antiinflammatory effects of budesonide in human fetal lung. Am J Respir Cell Mol Biol 2016;55: 623-32. 